\title{
Linear-array apertures for in-flight dynamic solar calibration of radiometric channels for Earth radiation-budget applications
}

\author{
Nour E. Tira, J. R. Mahan, Robert B. Lee III, and R. J. Keynton
}

\begin{abstract}
The zero-frequency gain of nonimaging radiometers used in Earth radiation-budget applications is usually verified by a procedure that allows the instrument to view the Sun through an appropriate attenuating aperture and then equates its response to the known attenuated solar constant. However, channel intercomparison often requires that data from a low-resolution, relatively slow instrument such as an active-cavity radiometer be compared with data from a high-resolution, fast instrument such as a scanning thermistor-bolometer radiometer. In such a case, consideration of the difference in the dynamic responses of the two channels may be important. A novel technique for in-flight measurement of the radiometric transfer function of such instruments is described and then demonstrated through the use of a high-order dynamic model of the total, wide-field-of-view, nonscanning channel of NASA's Earth Radiation Budget Experiment (ERBE).
\end{abstract}

\section{Introduction}

The Earth Radiation Budget Experiment (ERBE) is primarily intended to address the global-warming issue through long-term monitoring of the net planetary radiative balance. ERBE consists of a suite of nonimaging radiometric channels carried on three satellites: the Earth radiation-budget satellite (ERBS) and the National Oceanic and Atmospheric Administration's (NOAA's) satellite NOAA-9, which was launched in 1984, and the NOAA-10 satellite, which was launched in 1986. Each satelite carries four nonscanning active-cavity radiometers and three scanning thermistor-bolometer radiometers. The nonscanning instruments cover two fields of viewwide and medium - and two wavelength intervalsvisible and total. The scanning channels are narrowfield-of-view instruments covering three wavelength intervals-visible, longwave, and total. Static inflight calibrations of all channels is performed through on-board sources and appropriately attenuated views of the sun. The interested reader is referred to Refs.

N. E. Tira and J. R. Mahan are with the Department of Mechanical Engineering, Virginia Polytechnic Institute and State University, Blacksburg, Virginia 24061; R. B. Lee III and R. J. Keynton are with the Radiation Sciences Branch, NASA Langley Research Center, Hampton, Virginia 23681.

Received 20 February 1992; revision received to January 1994. 0003-6935/94/245617-11\$06.00/0.

(C) 1994 Optical Society of America.
1-3 for detailed descriptions of these instruments and their in-flight static calibration.

The thermophysical characteristics of space-borne radiometers such as those carried by the ERBE satellites will invariably change with time. These changes occur because of degradation of optical surfaces that results from ultraviolet radiation, micrometer impact, outgassing, condensation of outgassed products, the presence of atomic oxygen, and chargedparticle flux from the sun. Also, electrical and thermal impedances and amplifier gains naturally drift with age and use. For this reason it is necessary to periodically measure the instrument transfer function in situ.

The zero-frequency gain of radiometric channels used in Earth radiation-budget applications is usually verified with a procedure that allows the instrument to view the Sun through an appropriate attenuating aperture and then equates its response to the known attenuated solar constant. By zero-frequency it is meant that the channel is assumed to be in a state of thermal and electrical equilibrium during calibration. However, channel intercomparison often requires that data from a low spatial resolution, relatively slowly responding instrument such as an activecavity radiometer be compared with data from a high spatial resolution, relatively fast instrument such as a scanning thermistor-bolometer radiometer. In this case, consideration of the difference in the dynamic responses of the two channels may be important. 
Described here is a novel technique for the measurement of the radiometric transfer function of such instruments during solar calibration. The technique exploits the fact that the variation with time of the radiative input and the channel output, both rich in harmonic content, are known during the solarcalibration event.

In the current article, numerical simulation is used to evaluate the proposed new in-flight dynamic solarcalibration technique. This approach requires the use of a high-order dynamic electrothermal model of a radiometric channel that is similar to channels for which the method is intended. Here, the adjective pair dynamic electrothermal refers to the fact that coupled electrical and thermal processes combine to convert the time-varying radiative input into a timevarying electrical output. Fortunately, such a model already exists for the ERBE total, wide-field-of-view, nonscanning channel and so did not have to be specially formulated for the current study. 4,5 The ability of this model to accurately predict the observed dynamic electrothermal behavior of the actual instrument during the solar-calibration event qualifies it to evaluate the proposed in-flight solarcalibration method. ${ }^{6,7}$

\section{Transfer Function of the ERBE Total, Wide-Field-of-View, Nonscanning Channel}

Operation of the ERBE nonscanning channels is based on the active-cavity radiometer (ACR) concept. An ACR consists of an electrically heated cavity that is thermally connected to a constant-temperature heat sink through a thermal impedance. The cavity is actively controlled to a constant-temperature difference above that of the heat sink by a servo-controlled electrical heater. During operation, as the radiativepower input to the cavity changes, the electricalpower input is automatically adjusted as required to maintain the set-point temperature difference. The required heater power is then taken as the instrument output. More complete descriptions of the ERBE nonscanning ACR channels may be found elsewhere. ${ }^{2,4-7}$

A dynamic electrothermal model of the ERBE total, wide-field-of-view, nonscanning instrument has been formulated in Refs. 4 and 5 and has been shown to be capable of accurate simulation of the solar-calibration event.6,7 The model is based on first principles and requires no adjustable constants to force it to conform to reality. Tens of thousands of rays are traced with the Monte-Carlo method to determine the distribution of the incident radiation on the cavity surface, and a finite-element formulation with hundreds of elements is used to determine the unsteady diffusion of this heat in the cavity walls and thermal impedance. The electronic circuit that controls the cavity's active heating is also correctly modeled, as described in Refs. 4 and 6. The Monte-Carlo and finite-element codes that were used were formulated specifically for this instrument.
The excellent agreement between the simulated and observed instrument transient responses reported in Ref. 7 establishes the validity of the dynamic electrothermal model for the current application. The first step then is to obtain the transfer function of this model so that transfer functions obtained later through the candidate in-flight dynamic-calibration technique can be compared with the known instrument dynamic-response characteristics.

Because temperature variations in the ACR are small—of the order of $1 \mathrm{~K}$ - the equations describing instrument behavior are essentially linear. Therefore, the principle of superposition may be applied with negligible error with respect to the input function. This permits the individual harmonic components of an input signal to be introduced one at a time, rather than through the introduction of a complex transient input signal. In this way we can obtain the transfer function throughout the frequency range of interest without encountering the problems, which are described later in this study, inherent in the introduction of a complex time series made up of many harmonic components.

A series of harmonic-input irradiance signals whose frequencies are within the range of the model's frequency response, or bandwidth, is introduced to the model. This is accomplished when input to the dynamic electrothermal model is a simulated blackbody source whose power output varies sinusoidally with a specified amplitude and mean value at a given frequency. Each such input function then has the form

$$
y_{i}(t)=A_{i}+B_{i} \sin (2 \pi f t),
$$

where $t$ is the time, $A_{i}$ is the mean value, and $B_{i}$ is the magnitude of the input function, whose frequency is $f$. Once steady-state conditions are attained, the equation of the output must be similar in form to that of the input:

$$
y_{0}(t)=A_{0}+B_{0} \sin \left(2 \pi f t+\theta_{0}\right) .
$$

Table 1. Theoretical Transfer Function of the ERBE Total, Wide-Fleld-of-Vlew, Nonscanning Channel

\begin{tabular}{cccc}
\hline $\begin{array}{c}\text { Frequency } \\
(\mathrm{Hz})\end{array}$ & Gain $^{a}$ & $\begin{array}{c}\text { 20Log } \log _{10}[\mathrm{Gain}] \\
(\mathrm{dB})\end{array}$ & $\begin{array}{c}\text { Phase } \\
(\mathrm{deg})\end{array}$ \\
\hline 0.001563 & 1.00532 & 0.04609 & -4.80 \\
0.003125 & 1.01752 & 0.15086 & -9.15 \\
0.012500 & 1.05700 & 0.48150 & -35.35 \\
0.025000 & 1.03264 & 0.27898 & -68.36 \\
0.033300 & 0.92940 & -0.63595 & -97.83 \\
0.040000 & 0.75660 & -2.42267 & -113.0 \\
0.050000 & 0.51980 & -5.68327 & -124.5 \\
0.066700 & 0.31185 & -10.1211 & -140.1 \\
0.100000 & 0.14134 & -16.9947 & -158.7 \\
0.200000 & 0.03643 & -28.7708 & -170.7 \\
0.400000 & 0.00957 & -40.3818 & -177.7 \\
\hline
\end{tabular}

${ }^{a} B_{i} / B_{0}$. 
A curve of the form of Eq. (2) is fitted to the steady-state output signal through the use of the method of least squares to determine the output parameters $A_{0}, B_{0}$, and $\theta_{0}$. Once these parameters have been obtained for a given frequency $f$, the channel gain at that frequency is given by $B_{0} / B_{i}$, and the phase lag is $\theta_{0}$.

Table 1 and Fig. 1 give the transfer function for the range of frequencies of interest. Note that there is a nearly constant gain of essentially zero decibels in the frequency range $0 \leq f \leq 0.03 \mathrm{~Hz}$. Across most of this frequency range the gain is actually slightly greater than zero, and this suggests a slightly underdamped second-order system. Beyond a frequency of approximately $0.03 \mathrm{~Hz}$, which may be assumed to be the corner frequency, the curve begins to decrease at a rate of $-40 \mathrm{~dB}$ decade. The phase angle ranges from $0^{\circ}$ to $-180 \mathrm{deg}$, with a phase angle of $-90 \mathrm{deg}$ at the corner (or natural) frequency $f_{n}$. Because these characteristics closely match those of a second-order system, it is convenient to interpret the system's dynamic response according to the known form of the
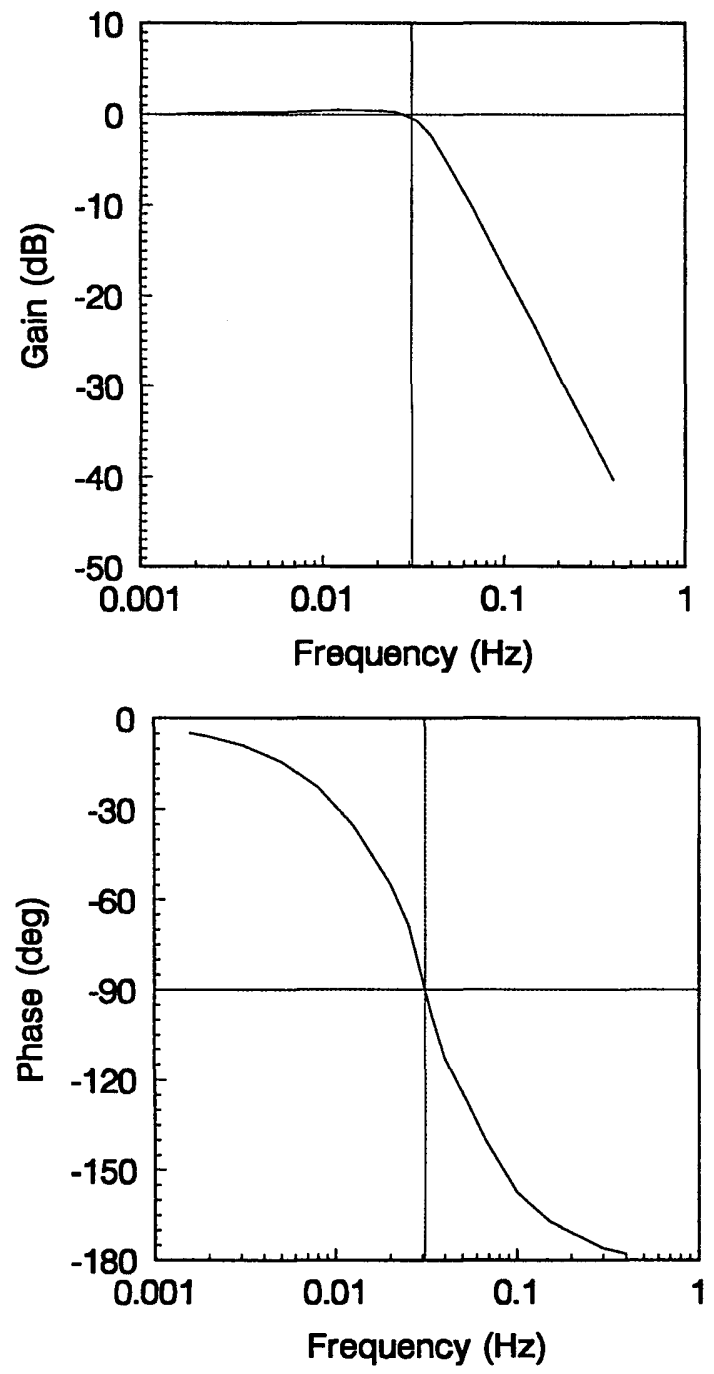

Fig. 1. ERBE total, wide-field-of-view, nonscanning channel transfer function (see Table 1): (a) gain and (b) phase angle.

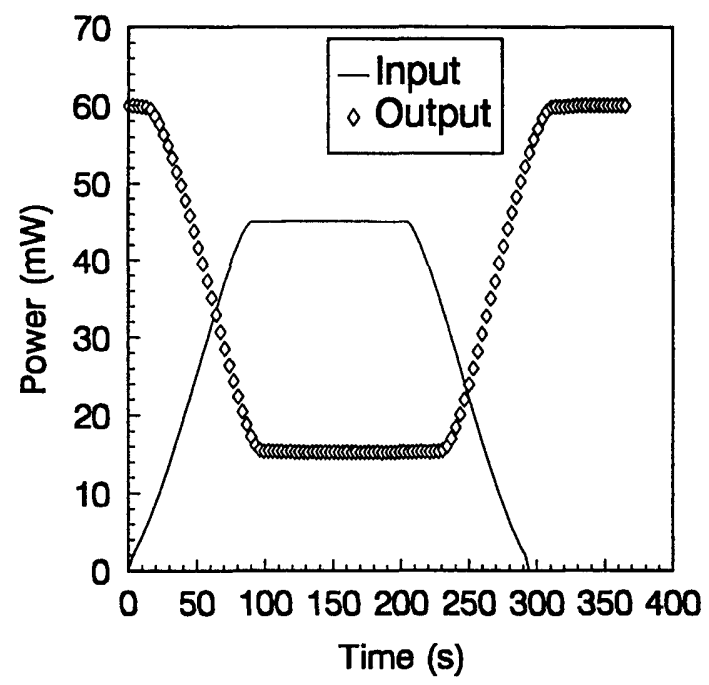

(a)

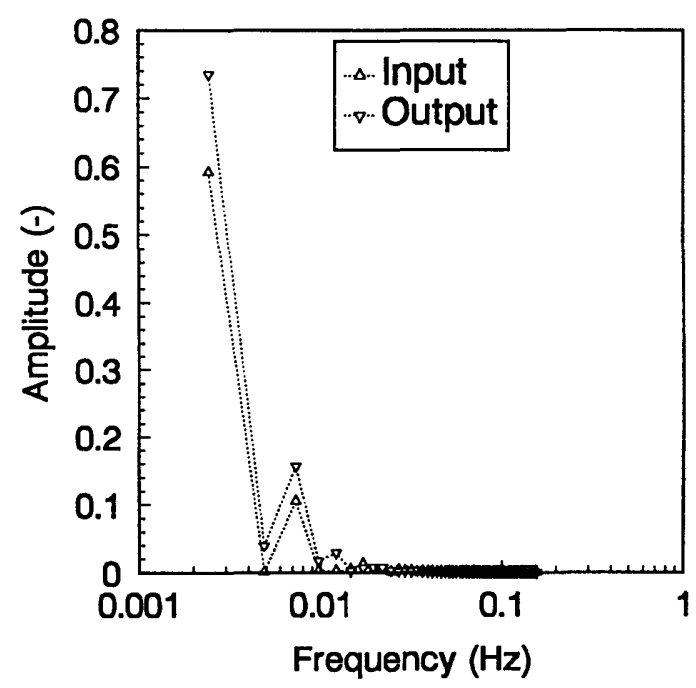

(b)

Fig. 2. Actual ERBS solar calibration of 28 December 1984: (a) theoretical input function (solid curve) and measured output data (diamonds), and (b) the corresponding frequency spectra.

transfer function for such systems:

$$
G(\omega)=\frac{1}{\left.1-\left(\omega / \omega_{n}\right)^{2}+2 i \zeta \omega / \omega_{n}\right)},
$$

where $\zeta$ is the damping ratio and $\omega_{n}=2 \pi f_{n}$ is the natural angular frequency.

The time constant $\tau$ for a second-order linear system can be defined as

$$
\tau=1 /\left(2 \zeta \omega_{n}\right) .
$$

Fitting Eq. (3) to the results in Table 1, we find that $f_{n}=\omega_{n} / 2 \pi=0.038 \mathrm{~Hz}$ and $\zeta=0.6$. With these values, a time constant of approximately $3.5 \mathrm{~s}$ is obtained. The electrical time constant of the ERBE ACR servo system is approximately $0.01 \mathrm{~s}$. Thus, it is clear that the frequency response of the ACR is limited by the diffusion of heat within the active cavity and the thermal impedance, and so $\tau$ can be 


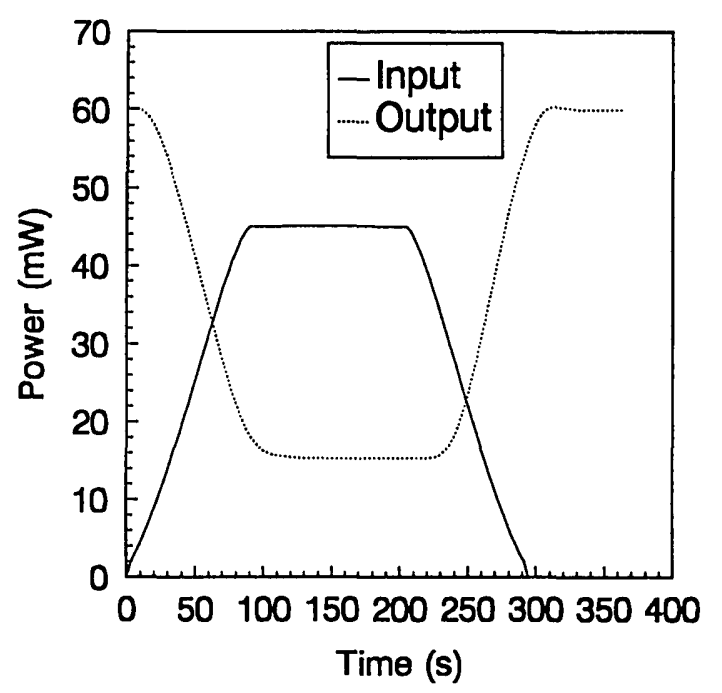

(a)

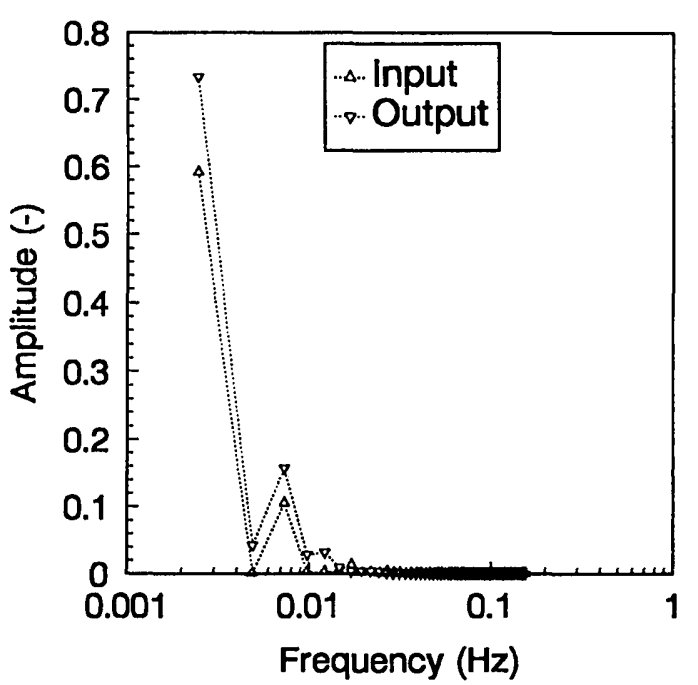

Fig. 3. Simulated ERBS solar calibration of 28 December 1984: (a) theoretical input function (solid curve) and simulated output data (dotted curve), and (b) the corresponding frequency spectra.

thought of as the thermal time constant of the instrument. It should be pointed out that without active heating the corner frequency would be much lower and the time constant much longer.

\section{Study of Actual and Simulated Solar-Calibration Data for the ERBE ACR}

The search for an in-flight dynamic solar-calibration technique began with an attempt to recover the transfer function of the ERBS total, wide-field-ofview, nonscanning channel from actual solar-calibration data. The input function associated with the solar-calibration event is not measured but may be deduced to a high degree of accuracy on the basis of the known solar constant, the known evolution with time of the satellite-Sun orientation, and the known instrument-aperture geometry. Of course, the corresponding output function is measured in the case of an actual solar-calibration event, but it can also be

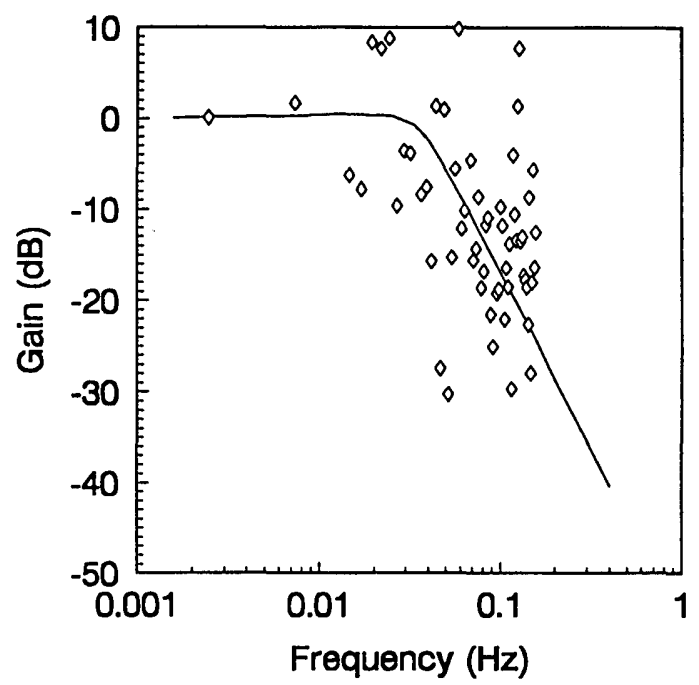

(a)

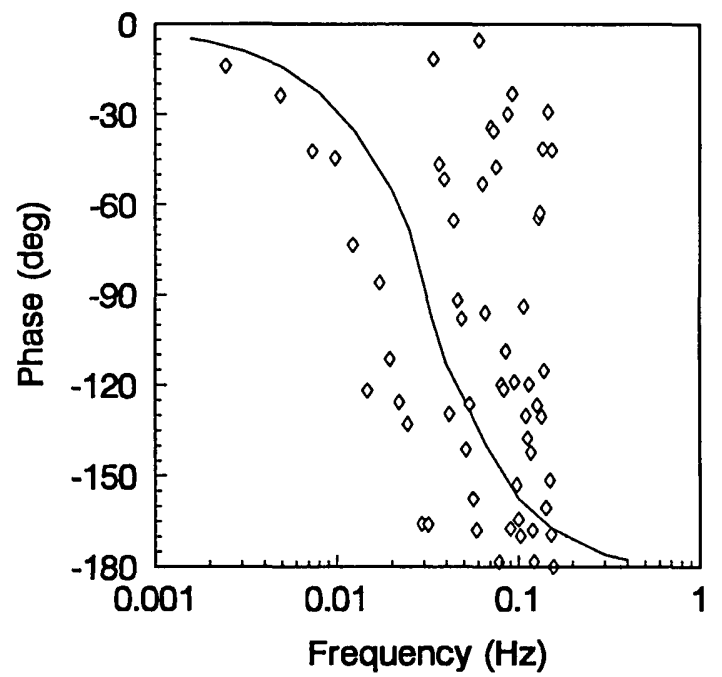

(b)

Fig. 4. Transfer function corresponding to the actual ERBS solar-calibration event of Fig. 2: (a) gain and (b) phase angle.

accurately simulated by use of the high-order electrothermal model described above.

The channel input and output time series and their corresponding frequency spectra for actual and simulated solar-calibration events are shown in Figs. 2 and 3 , respectively. These results correspond to the ERBS solar-calibration event of 28 December 1984. The frequency spectra are based on fast Fouriertransform analysis of the appropriate time series. As will be seen, it is significant that the spectra of Figs. 2 and 3 reveal that most of the energy is concentrated at low frequencies, below approximately $0.01 \mathrm{~Hz}$. The diamonds in Figs. 4 and 5 represent the transfer function based on the frequency spectra of Figs. 2 and 3, and the curves in Figs. 4 and 5 are the theoretical transfer function repeated from Fig. 1.

Extensive scatter is clearly seen in both transfer functions of Figs. 4 and 5, and this scatter indicates the need for a better in-flight instrument dynamiccharacterization technique. Still, it is somewhat encouraging that the actual and simulated solarcalibration data produce essentially the same degree 

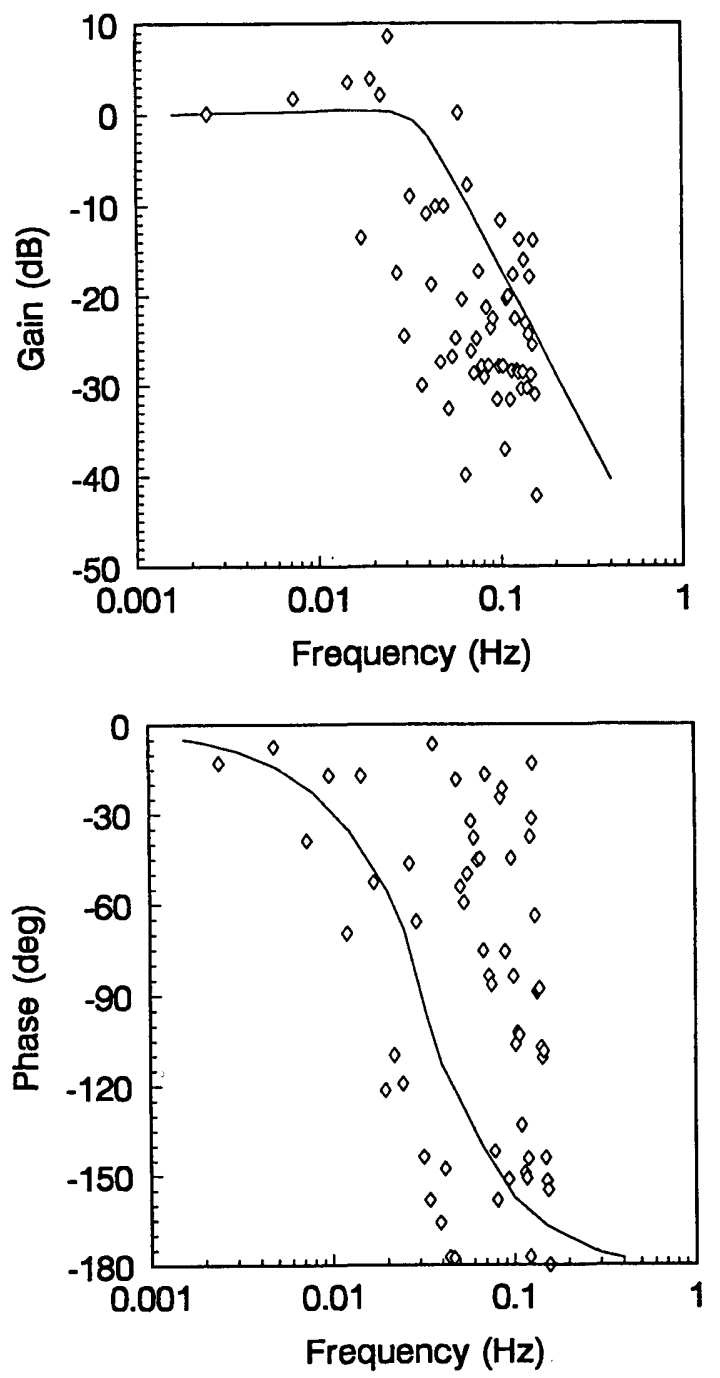

Fig. 5. Transfer function corresponding to the simulated ERBS solar-calibration event of Fig. 3: (a) gain and (b) phase angle.

of scatter and trends, and that the trends do seem to at least suggest the known transfer function. It would be possible in principle to improve the results if we were to perform averages on several sets of data. However, each solar-calibration event is unique and consists of only a single data set, and it is not possible in practice to perform several closely spaced solar calibrations that produce identical input time series.

After careful study of these results it became clear that the problem is due mostly to the fact that the signal energy is concentrated at low frequencies: the signal-to-noise ratio is simply too small at high frequencies to accurately recover the high-frequency spectral components. During solar calibration the ERBE nonscanning channels are rotated so that they view the Sun through a special circular field-of-view limiter called the solar port, and it is the relative motion of the Sun with respect to the solar port that determines the frequency distribution of the energy incident to the cavity during solar calibration. This suggests that perhaps other solar-port geometries might produce an input function during solar calibra-

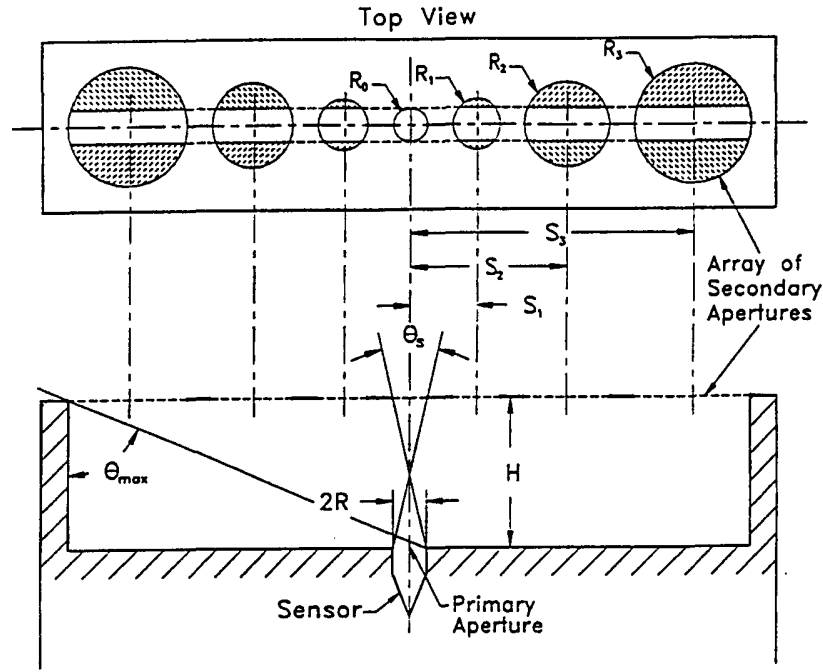

Fig. 6. Linear array of circular secondary apertures.

tion whose energy is more evenly distributed across the frequency spectrum of interest.

\section{Investigation of the Role of the Solar Port in Dynamic Solar Calibration}

It has already been demonstrated (see Section 3 ) that a simple circular aperture such as the ERBE solar port produces an input function whose energy is concentrated at low frequencies and thus is unsuitable for dynamic solar calibration. Further, results from the previous section suggest that a solar port that produces an input signal whose energy is more evenly distributed across the instrument band might solve the problem. In this section the effect of the solar-port geometry on its frequency-response characteristics is studied. In particular, two classes of solar-port geometry are investigated: a linear array of fixed-radius multiple apertures, and a linear array of varying-radius multiple apertures.

\section{A. Linear Array of Fixed-Radius Circular Apertures}

In the discussion that follows the solar port is also referred to as the secondary aperture to distinguish it from the primary aperture, or precision aperture, through which radiation actually enters the ACR cavity. The solar port, or secondary aperture, then acts as the field-of-view limiter during solar calibration. The first class of secondary apertures that we

Table 2. Dimensions of the Linear-Array Secondary-Apertures Configurations Studied

\begin{tabular}{|c|c|c|c|c|c|c|}
\hline Case & $R_{0} / R$ & S/R & $\mathrm{H} / \mathrm{R}$ & $\begin{array}{r}\theta_{\max } \\
(\mathrm{deg})\end{array}$ & $\begin{array}{l}\text { Number of } \\
\text { Secondary } \\
\text { Apertures }\end{array}$ & $\begin{array}{c}\text { Length } \\
L / 2 R\end{array}$ \\
\hline 1 & 2 & 6 & 10 & 80.00 & 15 & 44 \\
\hline 2 & 1 & variable & 20 & 74.24 & 13 & 70 \\
\hline 3 & 1 & variable & 20 & 51.40 & 9 & 24 \\
\hline 4 & 5 & variable & 60 & 85.66 & 15 & 789 \\
\hline
\end{tabular}



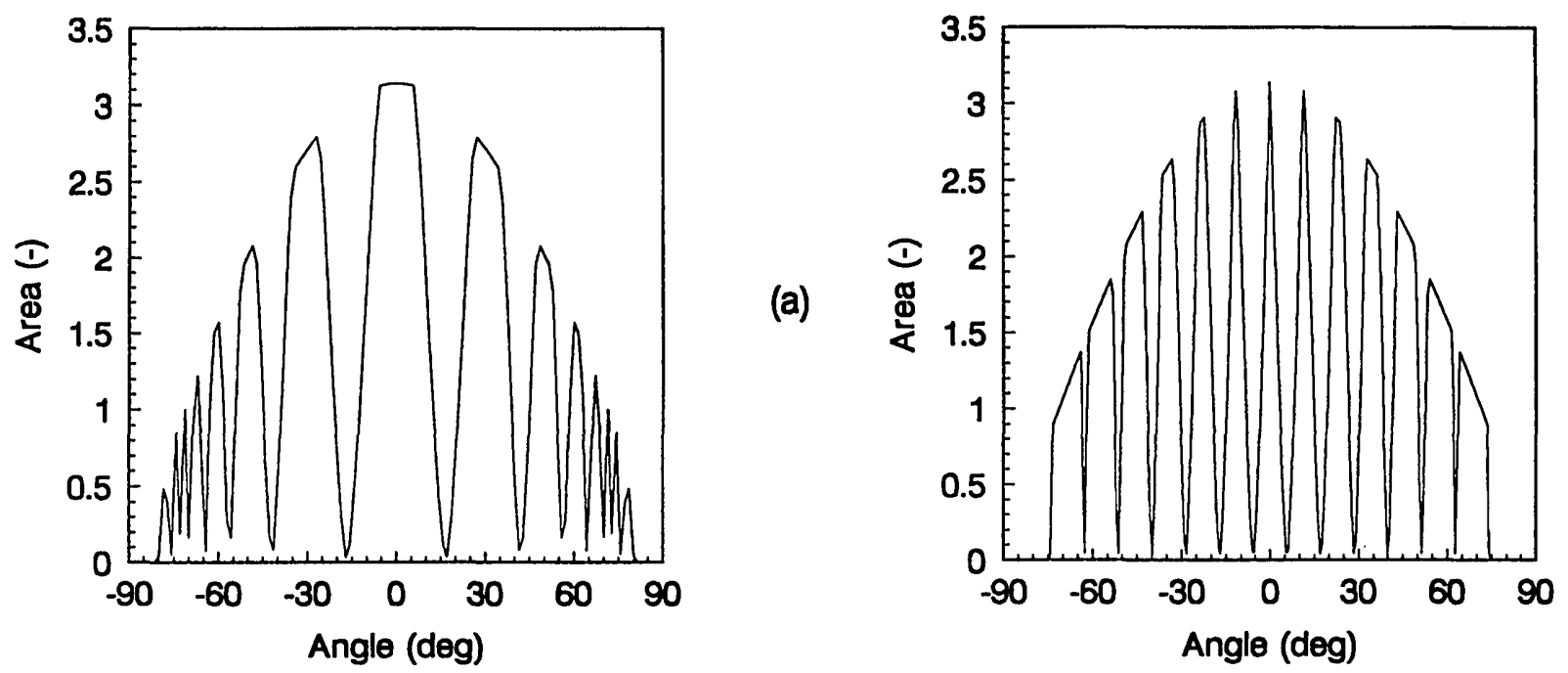

(a)
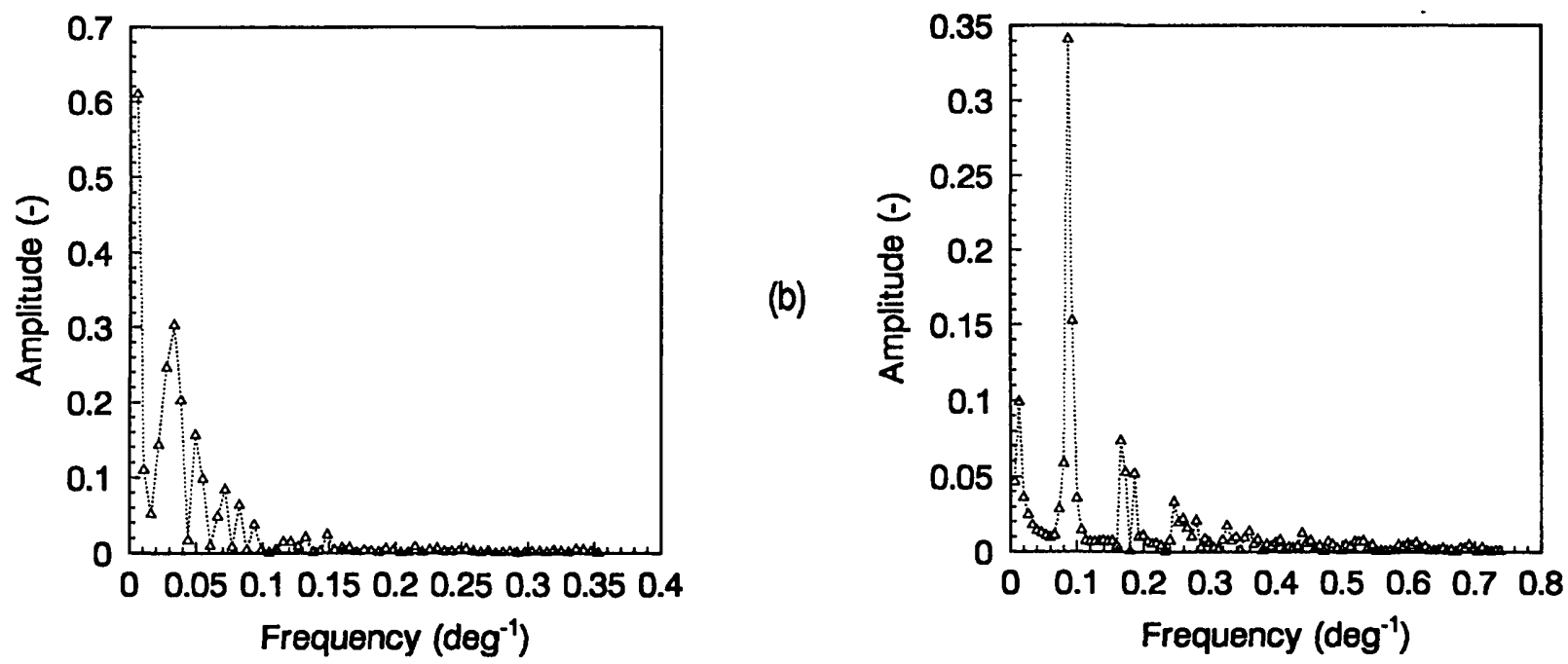

Fig. 7. (a) Theoretical input function produced by the fixedradius, linear-array aperture configuration of case 1 , and (b) the corresponding frequency spectrum (the triangles represent the calculated peaks of the Fourier components).

studied consists of a linear array of fixed-radius circular apertures similar to that shown in Fig. 6, except with $R_{0}=R_{1}=R_{2}=R_{3}$ and $S_{1}=S_{2}=S_{3}$. Although they are characterized as circular, the individual apertures making up the array could be truncated, as indicated by the striping in Fig. 6 . This is because the radiation entering the array through the striped area does not fall on the instrument's precision aperture.

For case 1 let the radii of the primary and secondary apertures be 1 and 2 units, respectively, and the height $H$ between them be 10 units. Further, let the fixed spacing $S$ between centers of adjacent secondary apertures be 6 units. The number of secondary apertures is set according to the maximum angle $\theta_{\max }$, beyond which no more direct radiation from the point source can enter the primary aperture. For the parameters given above, this maximum angle is 80 deg, and the number of secondary apertures is 15 . These dimensions are summarized in Table 2, which

Fig. 8. (a) Theoretical input function produced by the varyingradius, linear-array aperture configuration of case 2 , and (b) the corresponding frequency spectrum (the triangles represent the calculated peaks of the Fourier components).

gives the dimensions of the four secondary-aperture designs studied.

It is convenient to take as the theoretical input function the projected area of the secondary aperture on the primary aperture (the ACR precision aperture) as a function of the zenith angle. This projected area, which is generally the intersection of two ellipses, is proportional to the radiation input from a point source at a given zenith. This approach makes the analysis independent of the variation from one solar calibration to the next that occurs as a result of the finite size of the solar disk, but it does not in any way compromise the generality of the results. The frequency corresponding to the Fourier transform of such an input function has units of inverse degrees.

The theoretical input function corresponding to the secondary-aperture configuration of case 1 is shown in Fig. 7(a). Note that both the magnitude and the width of each lobe in Fig. 7(a) decrease with increasing zenith angles following the cosine law for pro- 


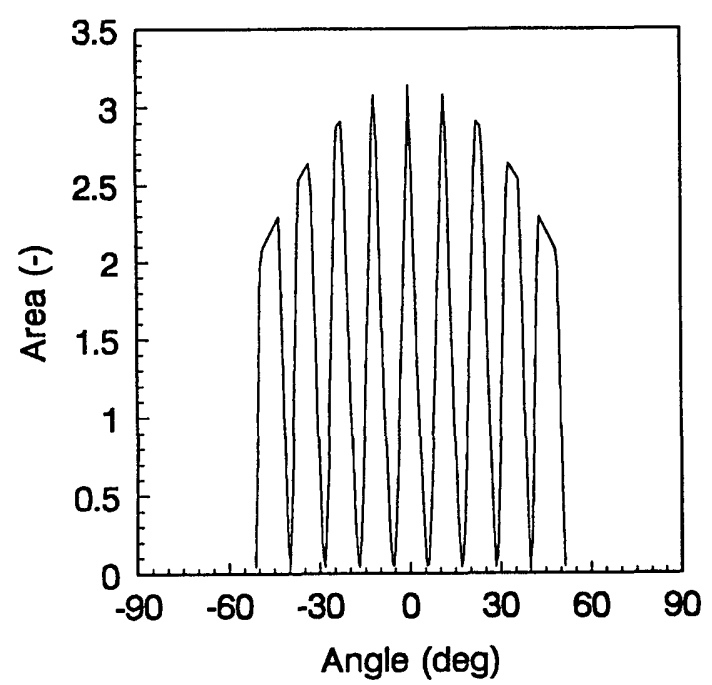

(a)

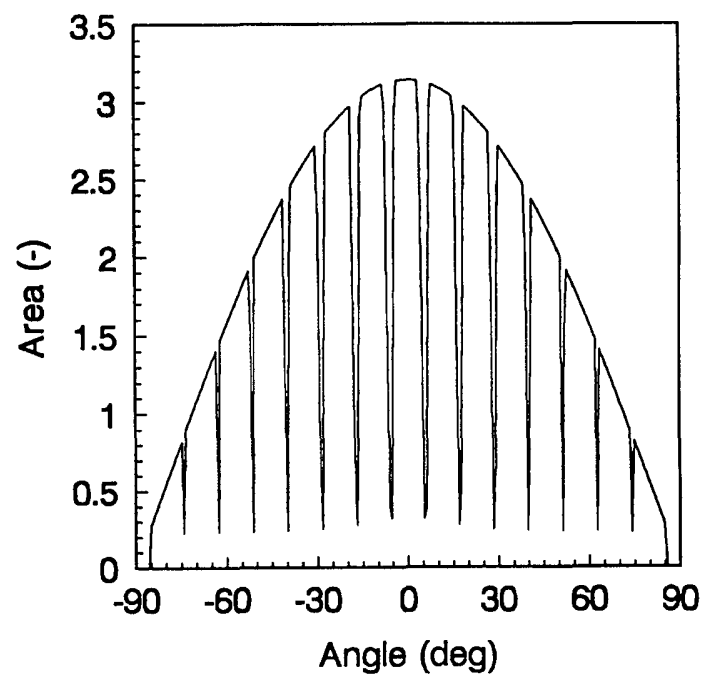

(a)
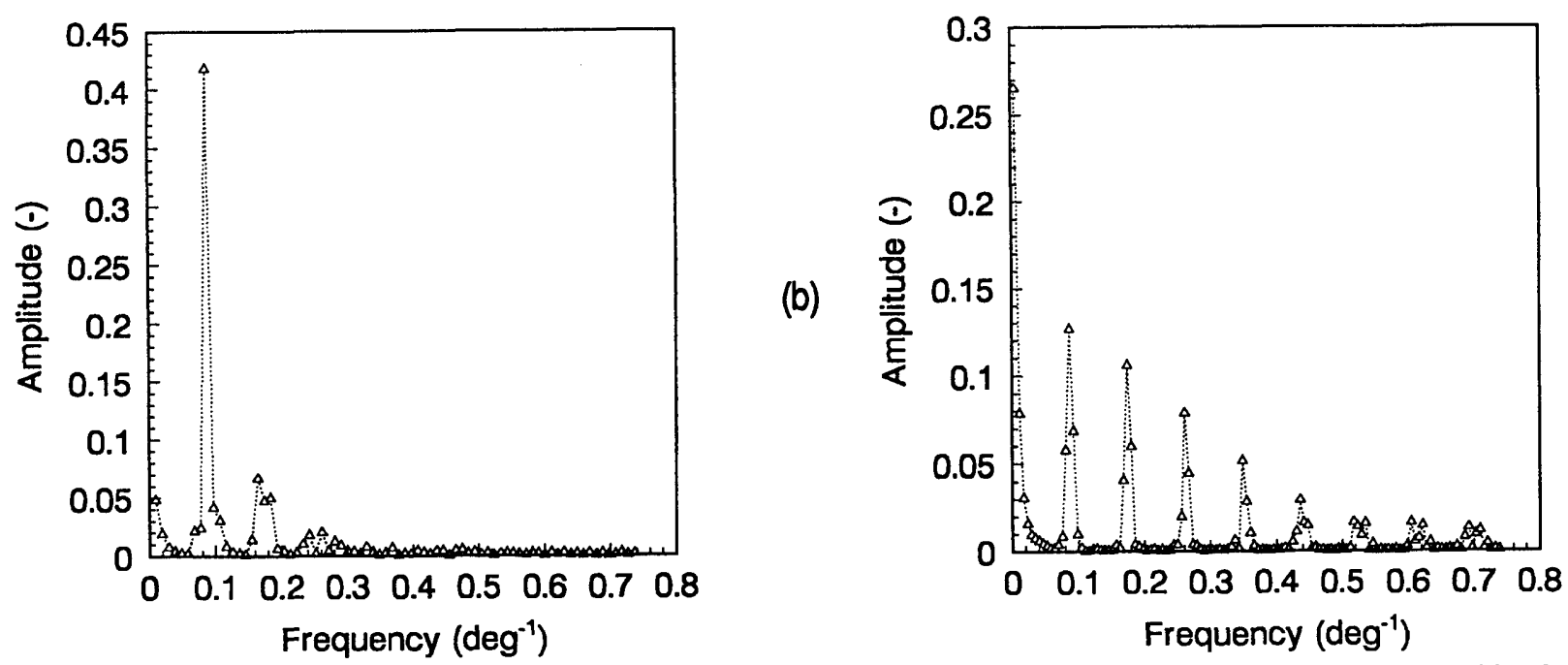

Fig. 9. (a) Theoretical input function produced by the varyingradius, linear-array aperture configuration of case 3 , and (b) the corresponding frequency spectrum (the triangles represent the calculated peaks of the Fourier components).

jected area. Figure $7(\mathrm{~b})$ verifies that the desired effect of the aperture array, which is to distribute more of the energy in the input signal to higher frequencies, has been achieved. The energy in the spectra of Figs. 2(b) and 3(b) is concentrated near a frequency (when converted to inverse angular units) of $0.0568 \mathrm{deg}^{-1}$, corresponding to the sample period of $16.7 \mathrm{deg}$, while the spectrum in Fig. 7(b) exhibits a series of secondary peaks of significant amplitude up to a frequency of approximately $0.17 \mathrm{deg}^{-1}$. The frequency of the first peak corresponds to the overall sample period, as it did in Figs. 2(b) and 3(b); however, there is now a second peak whose frequency corresponds to the period of the lobe centered at 0 deg, a third peak whose frequency corresponds to the period of the first side lobes, and so forth. Clearly, the idea of a solar port that consists of a linear array of secondary apertures looks promising for in-flight dynamic calibration, but one wonders if a fixed-radius array is the optimum geometry. Specifically, could

Fig. 10. (a) Theoretical input function produced by the varyingradius, linear-array aperture configuration of case 4 , and (b) the corresponding frequency spectrum (the triangles represent the calculated peaks of the Fourier components).

not the energy content, and thus the amplitude, of the harmonics be increased by an increase in the widths of the side lobes in the theoretical input function?

\section{B. Linear Array of Varying-Radius Circular Apertures}

The secondary-aperture arrays investigated in this section are similar to the array described in the previous subsection, except that now the secondaryaperture radii $R$ and the aperture spacings $S$ are allowed to increase, as shown in Fig. 6, in a way such that the width of each lobe in the time series is constant. We consider three aperture arrays for which the primary aperture $R_{s}$ is fixed at one unit, and the parameters $R_{0}$ and $H$ are varied. The number of apertures in the array and their radii and spacings are computed with a special computer program that ensures that all lobes in the resulting time series are of equal angular width. 


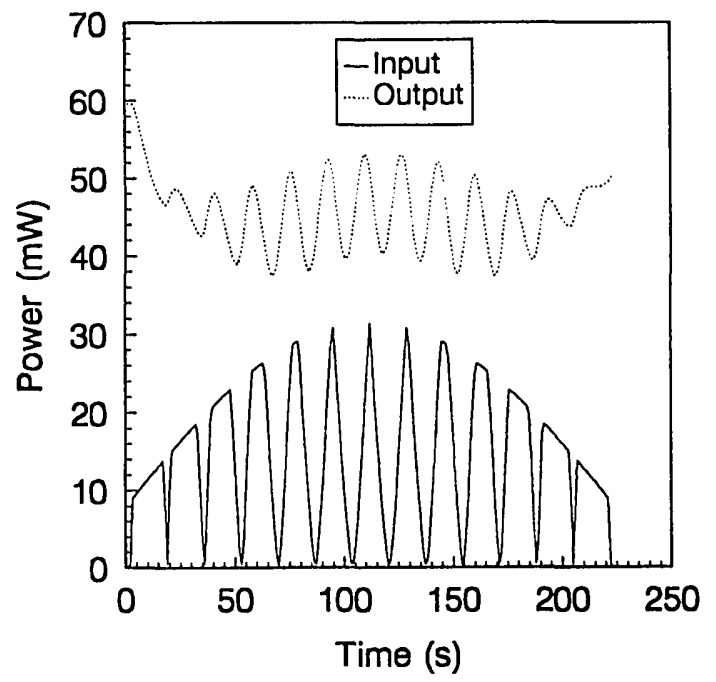

(a)

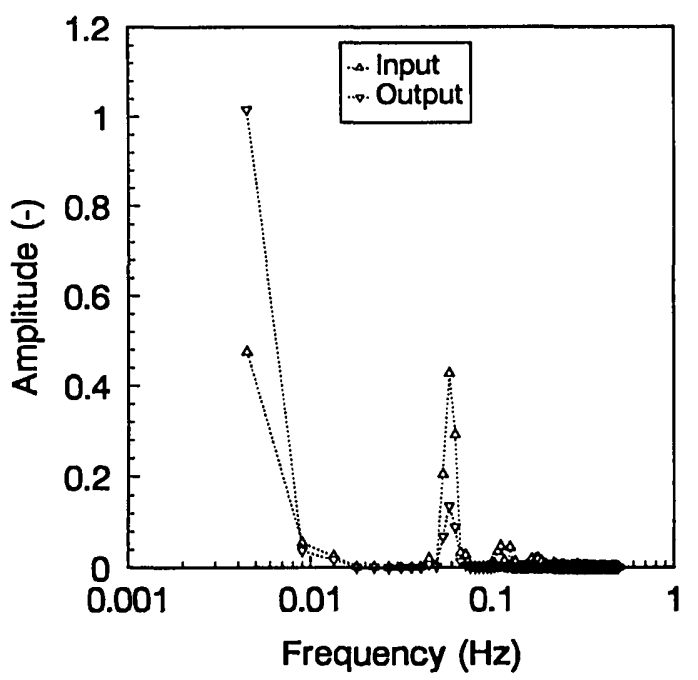

(b)

Fig. 11. (a) Radiative input signal and heater-power output produced by the linear-array secondary-aperture configuration of case 2 during simulated solar calibration of the ERBE total, wide-field-of-view, nonscanning channel, and (b) the corresponding frequency spectra.

Case 2 involves a linear array of 13 circular apertures. The central member of this array, which is also the smallest, has a radius $R_{0}$ that is equal to that of the primary aperture, and the spacing $H$ between the primary and secondary apertures is 20 primaryaperture radii. This is a truncated array in that it originally had 15 members, but one at each end has been suppressed.

Because the diameters of the individual members of the secondary array increase as the reciprocal of the cosine of the solar zenith angle, suppressing apertures at the ends of the array has a relatively large effect on the overall array length. Further, because the widths of all the lobes in the corresponding input function are equal while their heights decrease as the cosine of the zenith angle, the outlying lobes turn out to have relatively little influence on the measurement of the instrument transfer function. Thus, a large amount of space can be saved without a

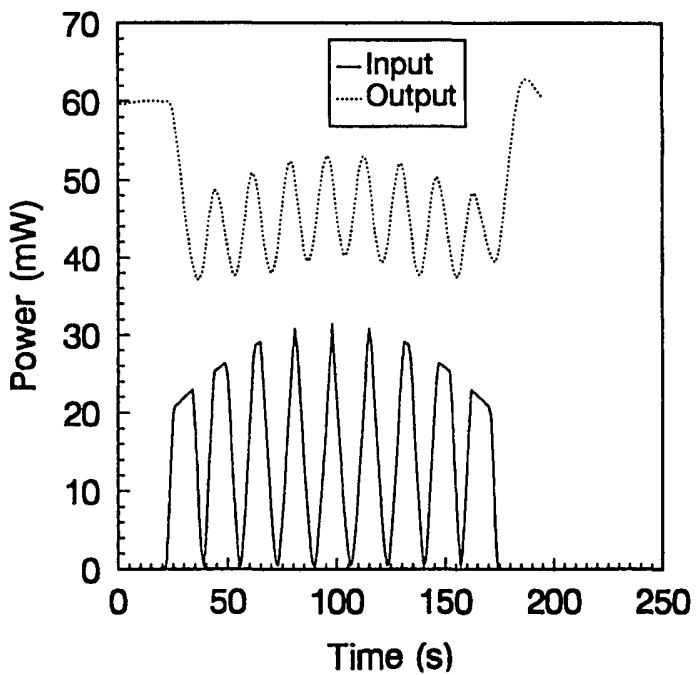

(a)

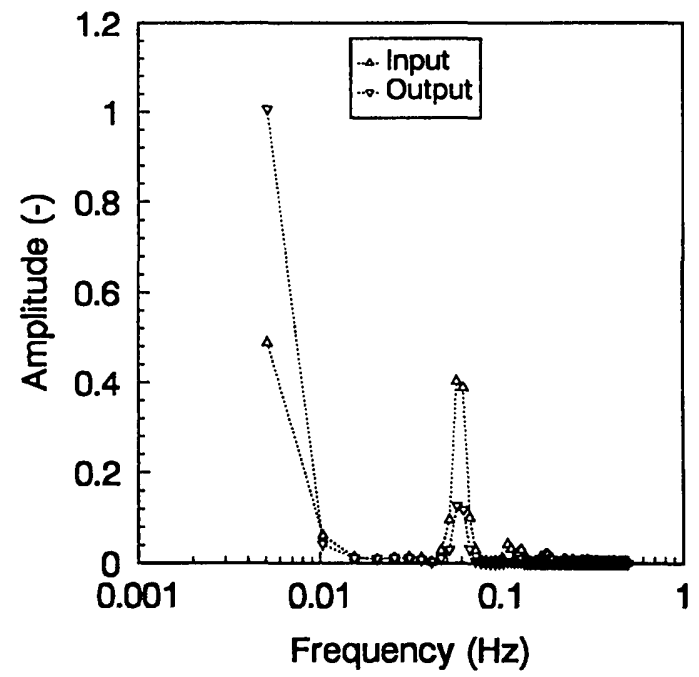

(b)

Fig. 12. (a) Radiative input signal and heater-power output produced by the linear-array secondary-aperture configuration of case 3 during simulated solar calibration of the ERBE total, wide-field-of-view, nonscanning channel, and (b) the corresponding frequency spectra.

significant loss of efficiency by the removal of outlying members of the array. However, the array in case 2 is still 70 primary-aperture diameters long. This means that if the primary aperture has a diameter of $10 \mathrm{~mm}$, which is representative of practical instruments, the array would be $0.7 \mathrm{~m}$ long. Case 3 is identical to case 2 except that now 6 of the original 15 members of the array have been suppressed, 3 at each end, which results in an array whose overall length is only 24 primary-aperture diameters. This array would be of practical size, but it is appropriate to ask if the ability of the array to recover the transfer function has been compromised by such extensive truncation.

Figure 8(a) shows the theoretical input function and Fig. 8(b) shows the corresponding frequency spectrum for the varying-radius aperture array of case 2. A comparison of the spectrum of Fig. 7(b) for the fixed-radius aperture array with that of Fig. 8(b) 


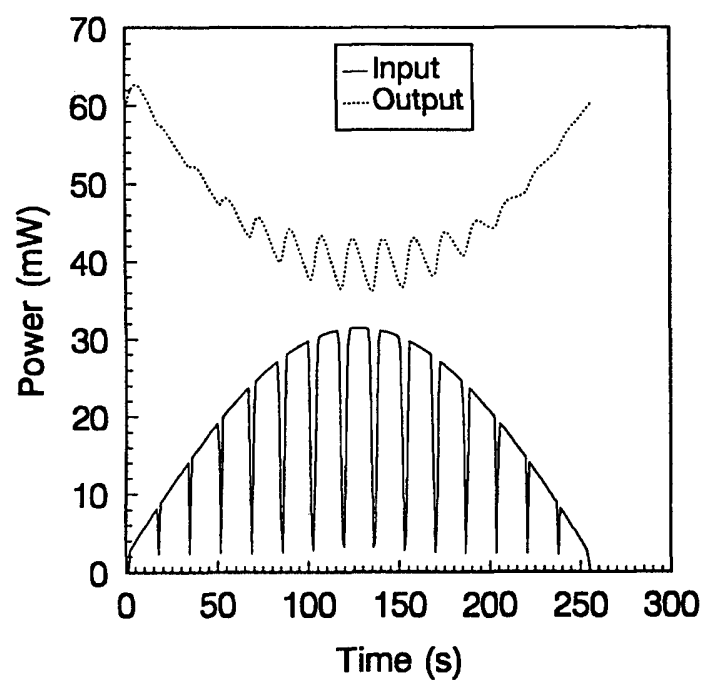

(a)

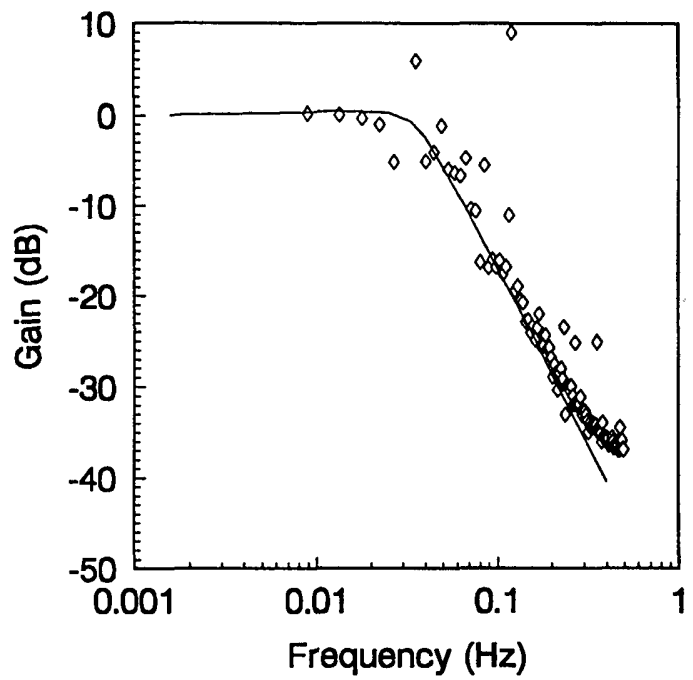

(a)
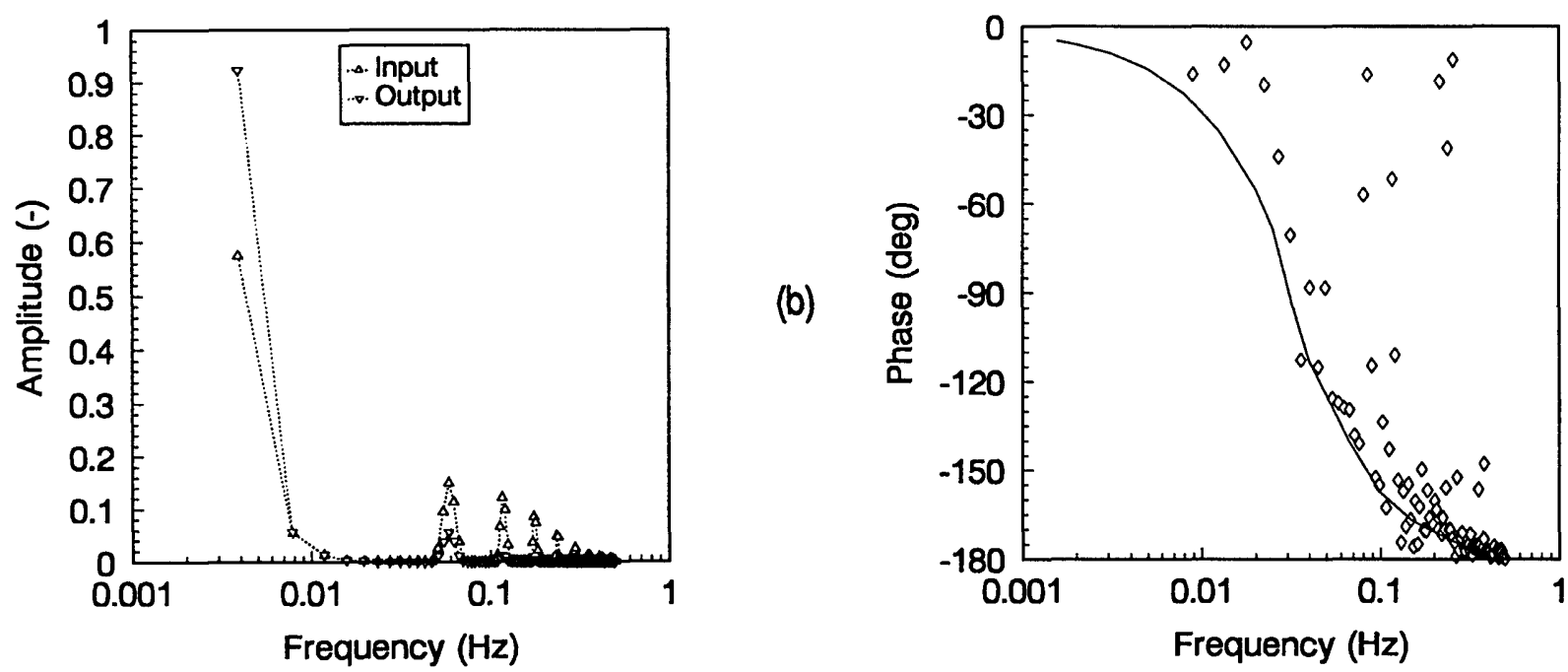

(b)

Fig. 13. (a) Radiative input signal and heater power output produced by the linear-array secondary aperture of case 4 during simulated solar calibration of the ERBE total, wide-field-of-view, nonscanning channel, and (b) the corresponding frequency spectra.

clearly shows that the input signal energy is distributed over a much wider frequency band in the latter than in the former: The highest-frequency harmonic that is distinguishable in Fig. 7(b) occurs at approximately $0.15 \mathrm{deg}^{-1}$, while in Fig. 8(b) a harmonic of comparable relative amplitude may be seen at approximately $0.45 \mathrm{deg}^{-1}$ Another difference between the two spectra is that in the case of the fixed-radius aperture array the largest peak in the spectrum corresponds to the fundamental, whereas in the case of the varying-radius array the largest peak is the harmonic associated with the central lobe of the time series.

Figures 9(a) and 9(b) show the theoretical input function and the corresponding spectrum for case 3 . It is clear from a comparison of Figs. 8(b) and 9(b) that the energy of the input function is not distributed as well across the frequency spectrum in case 3 as it is in case 2 . However, in view of the large reduction in the physical dimensions of the case-3

Fig. 14. Instrument transfer function (solid curves) for the ERBE total, wide-field-of-view, nonscanning channel as determined during simulated (diamonds) solar calibration with the linear-array secondary-aperture configuration of case 2: (a) gain and (b) phase angle.

aperture array, this bandwidth loss may be acceptable.

Case 4 is an array in which the radius $R_{0}$ of the central member is 5 times that of the instrument's primary aperture, the distance $H$ between the primary and secondary apertures is 60 primary-aperture radii, and there are 15 secondary apertures spanning a zenith-angle range of $85.66^{\circ}$. The overall width of this array is 789 primary-aperture diameters, which means that if the primary aperture is $10 \mathrm{~mm}$ in diameter, the array must be nearly $8 \mathrm{~m}$ long! The theoretical input function and the corresponding frequency spectrum for case 4 are shown in Fig. 10. Although the array of case 4 is clearly impractical from the point of view of overall size, an exhaustive parametric study failed to identify a linear array of a practical size that produced a better distribution of the input-function energy over its frequency spectrum. Thus, case 4 represents an attempt to establish some 

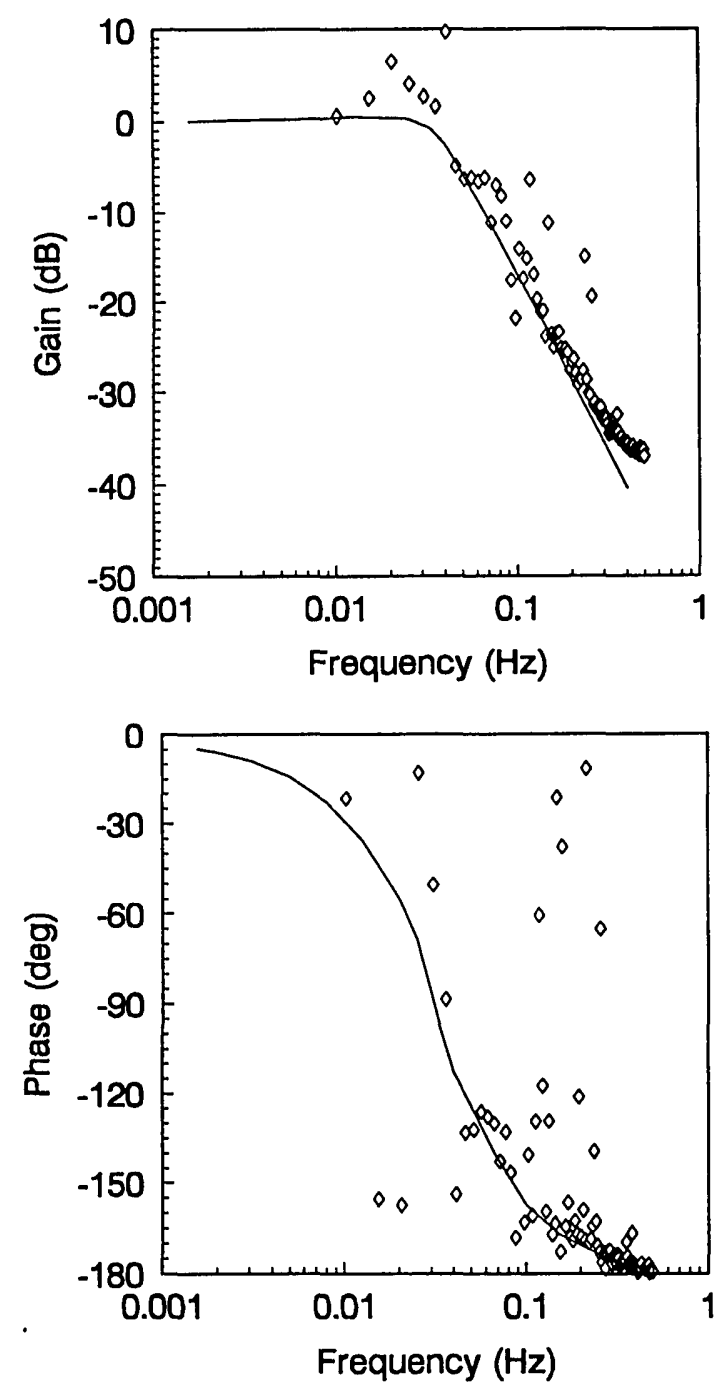

Fig. 15. Instrument transfer function (solid curves) for the ERBE total, wide-field-of-view, nonscanning channel as determined during simulated (diamonds) solar calibration with the linear-array secondary-aperture configuration of case 3: (a) gain and (b) phase angle.

measure of the ultimate potential of this class of secondary-aperture arrays.

C. Measurement of the Instrument Transfer Function with Secondary-Aperture Arrays

Cases 2, 3, and 4 have been used as the basis for the study of the ability of secondary-aperture arrays to recover the instrument transfer function. The theoretical input functions were transformed from their angular domains to the time domain, and the projected area was transformed into heat input that was scaled to that of the ERBE total, wide-field-of-view, nonscanning channel during solar calibration. The variations with time of the resulting heat input functions are shown in Figs. 11(a), , 12(a), and 13(a) for the three cases. When these input functions are introduced into the dynamic electrothermal model of the ERBE total, wide-field-of-view, nonscanning channel, the channel output in terms of electric-heater

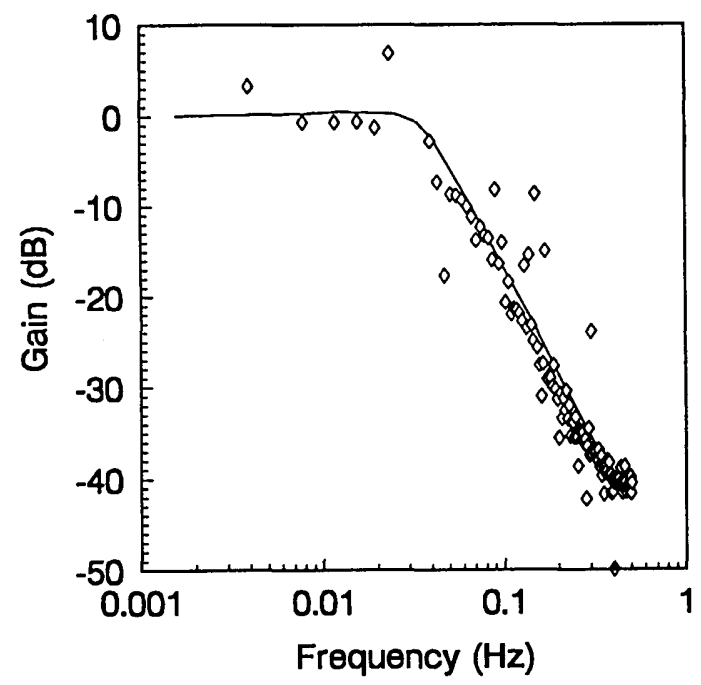

(a)

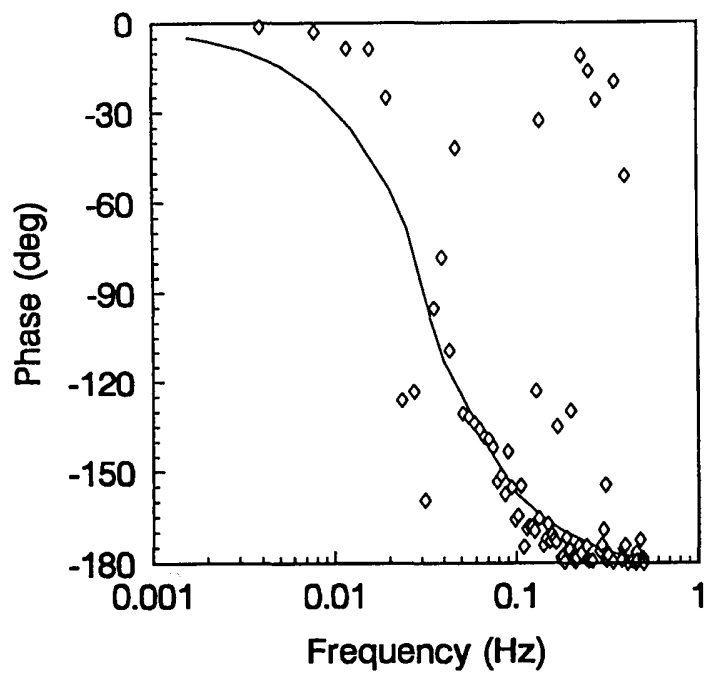

(b)

Fig. 16. Instrument transfer function (solid curves) for the ERBE total, wide-field-of-view, nonscanning channel as determined during simulated (diamonds) solar calibration with the linear-array secondary-aperture configuration of case 4: (a) gain and (b) phase angle.

power is as shown in Figs. 11(a), 12(a), and 13(a). The frequency spectra that correspond to the input and output functions are shown in Figs. 11(b), 12(b), and $13(\mathrm{~b})$. The instrument transfer functions corresponding to Figs. 11, 12, and 13 are shown in Figs. 14, 15 , and 16 , respectively. Also shown are the theoretical channel gain and phase shift already reported in Fig. 1. The agreement between the known transfer function and those obtained through dynamic simulation with the aperture arrays is remarkable. It is clear that the linear-array secondary-aperture configuration is vastly superior to a single secondary aperture for the recovery of the instrument transfer function from solar-calibration data. The most encouraging result, however, is that the practical design of case 3 recovers the channel transfer function almost as well as does the highly impractical design of case 4 . This latter is noticeably superior only at the highest frequencies (above $0.3 \mathrm{~Hz}$ ). 


\section{Summary and Conclusions}

The transfer function of the ERBE total, wide-field-ofview, nonscanning channel was accurately characterized through the use of the channel dynamic electrothermal model reported elsewhere. ${ }^{6,7}$ This theoretical result was then used as the basis for the evaluation of several candidate in-flight, dynamic solar-calibration methods. First, an attempt was made to recover the channel transfer function from actual and simulated solar-calibration data. The results that were obtained are almost identical, but they show unacceptable scatter about the known channel transfer function. It was deduced that the concentration of the input signal energy at low frequencies produced a low signal-to-noise ratio at high frequencies. This led to consideration of two different linear-array secondary-aperture geometries, one featuring fixed-radius apertures and the other featuring varying-radius apertures. The varying-radius design was shown to do a better job of distributing the available input energy more evenly across the frequency spectrum. Finally, it was demonstrated that a varying radius linear array secondary-aperture configuration of practical dimensions is capable of the accurate recovery of the transfer function of the ERBE total, wide-field-of-view, nonscanning channel from solar-calibration data.

The results of this study are encouraging. While no claim is made that the specific designs reported here are in any way optimal, it must be concluded that linear secondary-aperture arrays of the general class that was studied are sufficiently promising to be considered for future Earth radiation-budget instruments.

The authors are indebted to the Radiation Sciences Branch of the Atmospheric Sciences Division at NASA's Langley Research Center for its support of this work under grants NAG1-1016 and NAG11167.

\section{References}

1. B. R. Barkstrom, "The Earth Radiation Budget Experiment (ERBE)," Bull. Am. Meteorol. Soc. 65, 1170-1180 (1984).

2. M. R. Luther, J. E. Cooper, and G. R. Taylor, "The Earth Radiation Budget Experiment nonscanning instrument," Rev. Geophys. Space Phys. 24, 391-399 (1986).

3. L. P. Kopia, "Earth Radiation Budget Experiment scanner instrument," Rev. Geophys. Space Phys. 24, 400-406 (1986).

4. N. E. Tira. "Dynamic simulation of solar calibration of the total, Earth-viewing channel of the Earth Radiation Budget experiment (ERBE)," M. S. thesis (Department of Mechanical Engineering, Virginia Polytechnic Institute and State University, Blacksburg, Va., 1987).

5. N. E. Tira, "A study of the thermal and optical characteristics of radiometric channels for Earth radiation budget applications," Ph.D. dissertation (Department of Mechanical Engineering, Virginia Polytechnic Institute and State University, Blacksburg, Va., 1991).

6. J. R. Mahan, N. E. Tira, R. B. Lee III, and R. J. Keynton, "Comparison of the measured and predicted response of the Earth Radiation Budget Experiment active cavity radiometer during solar observations," Appl. Opt. 28, 1327-1337 (1989).

7. N. E. Tira, J. R. Mahan, and R. B. Lee III, "A dynamic electrothermal model for the ERBE nonscanning radiometer with application to solar observations and thermal noise," Opt. Eng. 29, 351-358 (1990). 